\title{
First double-detector results from Double Chooz experiment
}

\author{
Tsunayuki Matsubara* on behalf of Double Chooz collaboration \\ Tokyo Metropolitan University \\ E-mail: matsubara@hepmail.phys.se.tmu.ac.jp
}

\begin{abstract}
We report preliminary results of first $\theta_{13}$ measurements from the Double Chooz experiment with double-detector setup. In addition to the Far detector operation at $\sim 1 \mathrm{~km}$ baseline since 2011, we started the Near detector operation at $\sim 400 \mathrm{~m}$ baseline since beginning 2015 , which allows us to reach significantly improved sensitivity of the neutrino mixing angle $\theta_{13}$. From oscillation analysis using rate and spectral shape, we obtained $\sin ^{2} 2 \theta_{13}=0.111 \pm 0.018$ with $\chi^{2}$ /ndf $=$ $128.8 / 120$, combining 1 year of double-detector and 2 years of single-detector data. We also reported current efforts to reach better sensitivity with improved analysis as well as other physics programs.
\end{abstract}

38th International Conference on High Energy Physics 3-10 August 2016

Chicago, USA

${ }^{*}$ Speaker. 


\section{Introduction}

The neutrino oscillation parameters in three flavor scheme are three neutrino mixing angles $\left(\theta_{12}, \theta_{13}, \theta_{23}\right)$, three mass squared differences $\left(\Delta m_{21}^{2}, \Delta m_{31}^{2}, \Delta m_{32}^{2}\right)$ and one CP violation phase $\left(\delta_{C P}\right)$. Double Chooz is reactor neutrino experiment designed for precise measurement of the neutrino mixing angle $\theta_{13}$. The first non-zero indication of the $\theta_{13}$ from a reactor experiment was provided by Double Chooz in 2011 [1]. A robust observation of the $\theta_{13}$ was followed in 2012 by the Daya Bay and RENO experiments with multiple detectors [2,3]. The final precision of the $\theta_{13}$ by reactor experiment is a critical matter for current and future experiments addressing the possible observation of leptonic CP violation in neutrino oscillations, such as T2K and NOvA. Therefore, validation with multiple experiments is essential.

Reactor neutrino experiments use reactor power plant as a free and rich electron antineutrino source. A liquid-scintillator detector is located at $1 \sim 2 \mathrm{~km}$ baseline for direct measurements of $\sin ^{2} 2 \theta_{13}$, where maximum oscillation can be observed according to the survival probability; $\mathrm{P}\left(\bar{v}_{e} \rightarrow \bar{v}_{e}\right) \simeq 1-\sin ^{2} 2 \theta_{13} \sin ^{2}\left(1.27 \Delta \mathrm{m}_{e e}^{2} \mathrm{~L} / \mathrm{E}\right)$. We uses inverse beta-decay (IBD) process to detect electron antineutrinos. The electron antineutrino from reactor interacts with free proton in the detector and emits positron and neutron. The positron makes prompt signal by ionization and annihilation with electron, creating $1 \sim 8 \mathrm{MeV}$ energy deposit. As the energy is related to neutrino energy with $\mathrm{E}_{\text {prompt }}=\mathrm{E}(\text { kin. })_{e^{+}}+2 \mathrm{~m}_{e} \simeq \mathrm{E}_{\bar{v}_{e}}-\left(\mathrm{M}_{n}-\mathrm{M}_{p}\right)+\mathrm{m}_{e} \simeq \mathrm{E}_{\bar{v}_{e}}-0.782 \mathrm{MeV}$, measuring energy spectrum allows us to use spectral shape distortion with respect to oscillation probability. The neutron is captured by Gadolinium $(\mathrm{Gd})$ or Hydrogen $(\mathrm{H})$ and makes delayed signal with $\sim 8$ (2.2) $\mathrm{MeV}$ energy deposit and an time interval of $\tau \sim 30$ (200) $\mu$ sec for Gd (H) capture. Background is strongly suppressed by requiring time coincidence of those signals, which is well known as delayed coincidence technique. In addition, systematic uncertainties are further reduced by placing an identical detectors with shorter baseline.

Double Chooz is a pioneer experiment to measure the $\theta_{13}$. We reported single-detector $\theta_{13}$ results with improved analysis [4], first $\theta_{13}$ results using Hydrogen capture signal [5], and spectral distortion around $\mathrm{E}_{v}=5 \sim 7 \mathrm{MeV}$ [6]. In this article, we report preliminary results of first $\theta_{13}$ measurements from the Double Chooz experiment with double-detector setup.

\section{Double Chooz detectors}

Double Chooz detectors are located in Chooz (France) around two reactor cores operated by Électricité de France (EDF) with thermal power of 4.27 GWth for each cores. The Far detector (FD) has been operated since 2011 at $\sim 1 \mathrm{~km}$ baseline from reactor cores and $\sim 300$ m.w.e. depth. We started an operation of the Near detector (ND), which has identical structure with the FD, in begging 2015 at $\sim 400 \mathrm{~m}$ baseline and $\sim 120 \mathrm{~m}$.w.e. depth. In the single detector (SD) phase, we used experimental results provided by Bugey- 4 experiment as an anchor of neutrino flux reducing its error with about $1.7 \%$. In the double-detector (DD) setup, we can suppress the neutrino flux error with less than $0.1 \%$ thanks to nearly iso-flux setup of the ND and FD. Furthermore, identical detector structure can cancel correlated systematics like detection efficiency.

Double Chooz detector consists of main detector, called the Inner detector (ID), other detectors for background tagging and veto like the Inner veto (IV) and the Outer veto (OV). Figure 1 shows 
schematic view of the Double Chooz detector. The size of the detector is roughly $7 \mathrm{~m}$ in width and height. The ID has three cylindrical layers. Innermost region in $8 \mathrm{~mm}$ thickness of an acrylics vessel is called $v$-target. It is filled with $10.3 \mathrm{~m}^{3}$ of Gd-loaded $(1 \mathrm{~g} / \mathrm{l})$ liquid scintillator and used for Gd-capture region. The NT is surrounded by the $\gamma$-catcher region with $12 \mathrm{~mm}$ thickness of an acrylics vessel. This regions is filled with $22.3 \mathrm{~m}^{3}$ of liquid scintillator and used to detect escaped $\gamma$-ray and also used for Hydrogen-capture region. Those regions are surrounded by the Buffer region filled with $110 \mathrm{~m}^{3}$ mineral oil. On the stainless steel wall, total 390 low-background 10-inch PMTs are equipped.

The IV are optically separated with the ID and filled with liquid scintillator. Scintillation light is detected by 78 8-inch PMTs equipped on the wall and used for tagging and veto of background coming from outer detector. The OV are placed on those detectors. It consists of plastic scintillator strip, wavelength shifting fiber and multi-anode PMT. Some calibration systems are also equipped. LED systems are embedded in the ID and IV. Radioactive sources can be placed in the $v$-target and $\gamma$-catcher regions by two calibration deployment systems.

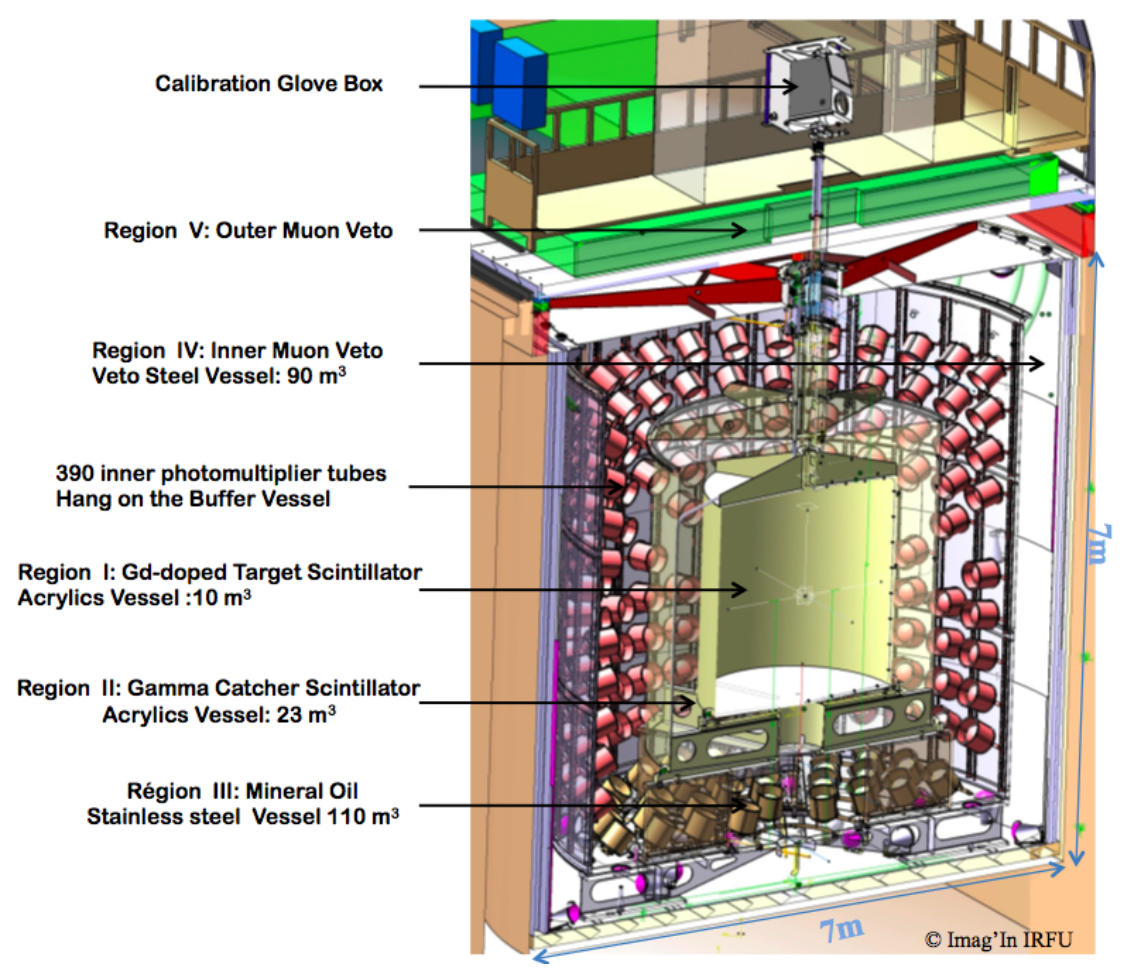

Figure 1: Schematic view of the Double Chooz detector

\section{Preliminary result with double-detector data}

We call FD data in the SD phase as FD-I. In the same way, FD and ND data in the DD phase as FD-II and ND, respectively. First double-detector analysis is performed using the FD-I, FD-II and ND data with 461.93, 212.21 and 150.76 days of live time. In addition, reactor-off data taken in the SD phase with 7.24 days of live time is also used for analysis to suppress background error. 
Our estimation for IBD and background rate in each period is summarized in Table 1. The IBD candidates were obtained with several steps of selection. We apply primary veto cuts for events induced by muon and light noise from PMT. Then delayed coincidence cut is applied for prompt and delayed energies as well as time and space correlation. Furthermore, additional veto cuts are also applied to reduce background events.

\begin{tabular}{|c|c|c|c|c|}
\hline & FD-I & Reactor off & FD-II & ND \\
\hline Live time (d) & "460.93 & 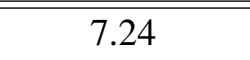 & 212.21 & 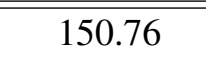 \\
\hline IIBD prediction $\left(d^{-1}\right)$ & 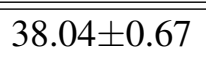 & 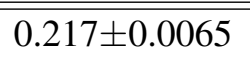 & $40.39 \pm 0.69$ & $280.5 \pm 4.7$ \\
\hline Accidental BG $\left(d^{-1}\right)$ & \multicolumn{2}{|c|}{$0070 \pm 0.003$} & $0.106 \pm 0.002$ & $0.344 \pm 0.002$ \\
\hline Fast-n + stop- $\mu\left(d^{-1}\right)$ & \multicolumn{3}{|c|}{$0.586 \pm 0.061$} & $3.42 \pm 0.23$ \\
\hline Cosmogenic BG $\left(d^{-1}\right)$ & \multicolumn{3}{|c|}{$\left(0.97_{-0.16}^{+0.41}\right)$} & $(5.01 \pm 1.43)$ \\
\hline Total prediction $\left(d^{-1}\right)$ & $39.63 \pm 0.73$ & $1.85 \pm 0.30$ & $42.06 \pm 0.75$ & $289.3 \pm 4.9$ \\
\hline $\begin{array}{l}\text { IBD candidates }\left(d^{-1}\right) \\
\text { (Number of events) }\end{array}$ & $\begin{array}{c}37.64 \\
(17351)\end{array}$ & $\begin{array}{c}0.97 \\
(7)\end{array}$ & $\begin{array}{l}40.29 \\
(8551)\end{array}$ & $\begin{array}{c}293.4 \\
(44233)\end{array}$ \\
\hline
\end{tabular}

Table 1: IBD and background rate in each period.

Remaining backgrounds are accidental coincidence, fast neutron, stopping muon and $(\beta, \mathrm{n})$ emitter from spallation products like ${ }^{9} \mathrm{Li}$. Those are already well suppressed and precisely estimated from data analysis as found in the table. The prediction of IBD events is calculated by thermal power, fuel evolution, mean energy released per fission, mean cross-section per fission distance between reactor cores and detectors, and detection efficiency.

Improvements of systematic errors can be found in Figure 2. In the SD phase, we improved uncertainties and the neutrino flux error was therefore dominant (black line). In the DD phase now, the error is strongly suppressed by the ND. All systematic uncertainties are well below $0.4 \%$ in rate+shape analysis.

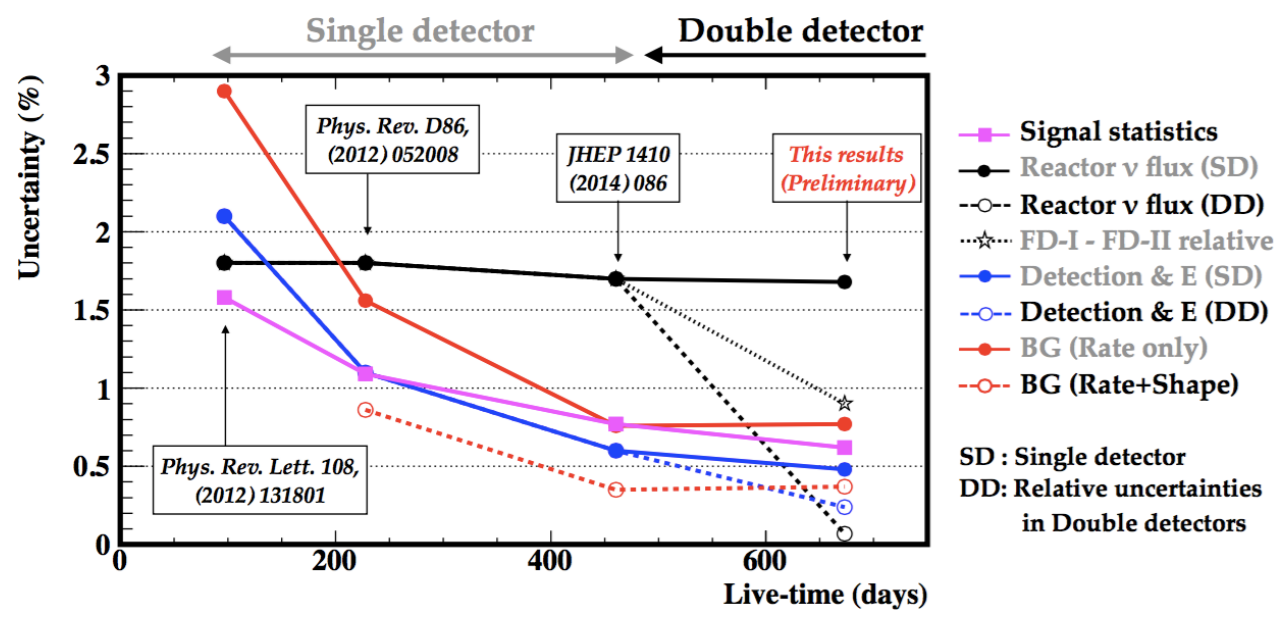

Figure 2: Improvements of systematic errors. 
Oscillation analysis has been done by fitting FD-I, FD-II and ND data simultaneously with predictions using both rate and spectral shape. We use the Chi-square method with systematic errors treated by pull term and covariance matrix. Correlation of systematic uncertainties between detector and periods are considered in the fit. Reactor-off period is also used for further background constraint.

As our preliminary results, we found the best-fit $\sin ^{2} 2 \theta_{13}=0.111 \pm 0.018$ with $\chi^{2}$ ndf $=$ 128.8/120. Non-zero $\theta_{13}$ is observed at 5.8 $\sigma$ C.L. Observed spectral ratio with the best-fit values are shown in Figure 3. As reported by our previous publication, we would see spectral distortion for all data set, especially in around $5 \mathrm{MeV}$ region. We will be able to validate the structure in neutrino flux model with more statistics.
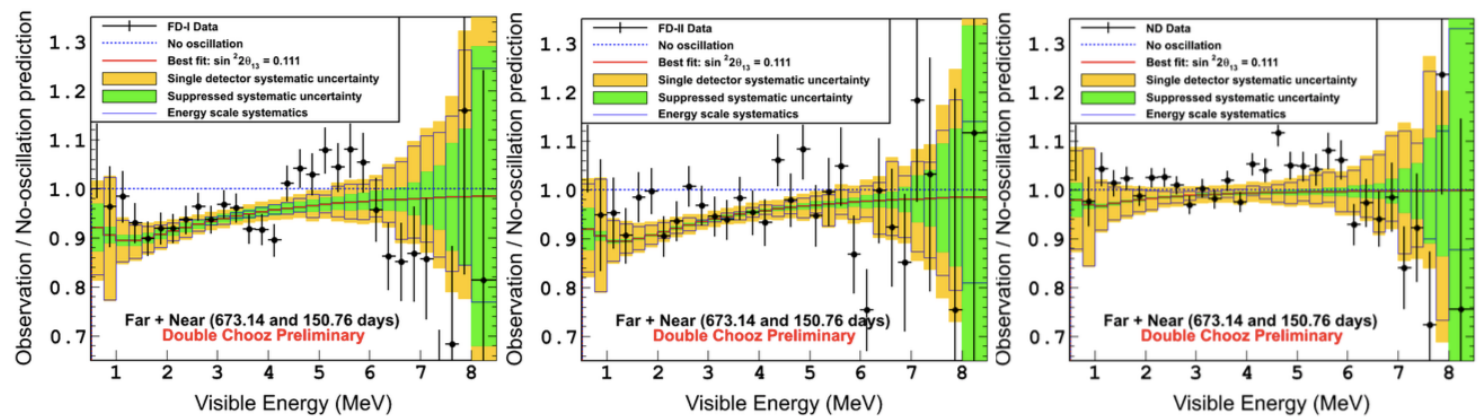

Figure 3: Observed spectral ratio with best-fit values.

\section{Future prospects}

Precise measurement of the $\theta_{13}$ by reactor experiments is still a key for current and future neutrino projects for $\mathrm{CP}$ violation and mass hierarchy. As we have small tension with other experiments, measurements with better precision is essential. Because current precision is limited by statistics as found in Figure 2, further improvements is expected. Expected sensitivity to the $\theta_{13}$ is shown in Figure 4. We can expect 0.01 level of precision with 5 years operation.

In order to get better precision earlier, we are considering to virtually increase our statistics by including Hydrogen-capture events, so called $\mathrm{Gd}+\mathrm{H}$ analysis. Advantage to include the events is larger volume as target. Three times of target volume is expected since the $\gamma$-catcher volume ( $\sim 20$ tons) can be used as target in addition to the $v$-target volume ( $\sim 10$ tons). Disadvantage of the $\mathrm{Gd}+\mathrm{H}$ analysis is an increase of accidental background by lowering delayed energy window to include Hydrogen captured peak $(\sim 2.2 \mathrm{MeV})$. Increase of systematic error is also challenging.

Status of Artificial Neural Network (ANN) development is reported as an analytical effort. We have developed ANN cut based on different distribution between signals and accidental background in delayed energy, correlation time and correlation distance. Thanks to the new cut we have succeeded to reduce the accidental background, which is almost negligible impact to the $\theta_{13}$ measurements. We also confirmed 2.5 times of IBD candidates than Gd-only analysis. The analysis results will be released soon ${ }^{1}$.

\footnotetext{
${ }^{1}$ New result with the $\mathrm{Gd}+\mathrm{H}$ analysis is released in September 2016 at CERN seminar.
} 


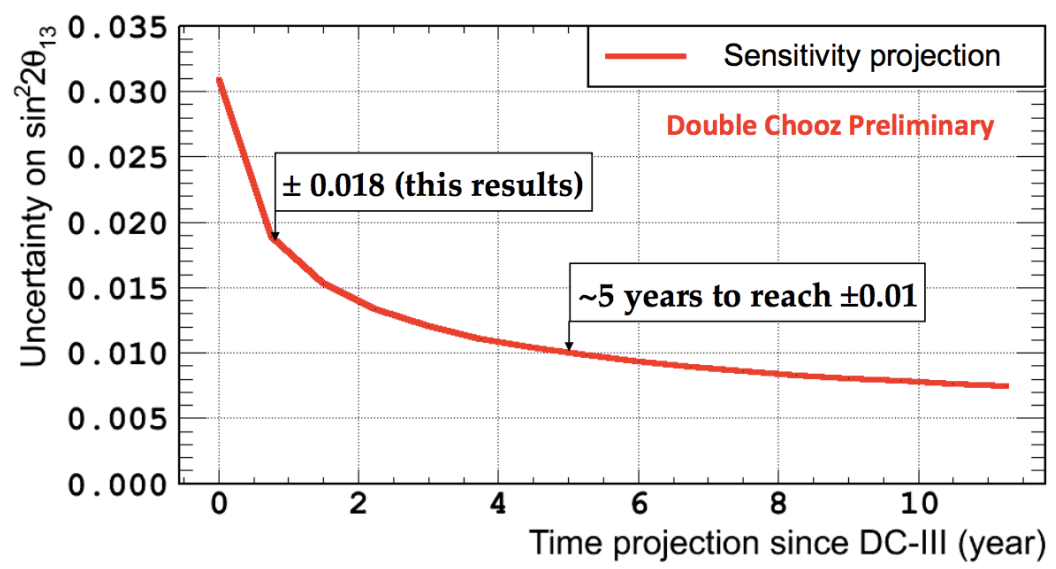

Figure 4: Expected sensitivity to the $\theta_{13}$.

We also reported about other physics programs. Neutrino directionality with liquid scintillator based detector is an interesting topic to obtain information on an orientation of neutrino source. We statistically measures the direction of incoming neutrinos using a vector from delayed to prompt signals. Sterile neutrino search is also hot topic that many experiments are trying to do. We showed expected sensitivity to test the hypothesis to light sterile neutrino with Double Chooz detectors located in $\sim 400 \mathrm{~m}$ and $\sim 1 \mathrm{~km}$ baseline.

\section{Sumamry}

Double Chooz experiment reported first results of preliminary $\theta_{13}$ measurement with doubledetector setup. The best-fit $\sin ^{2} 2 \theta_{13}$ is obtained as $\sin ^{2} 2 \theta_{13}=0.111 \pm 0.018$ with $\chi^{2} /$ ndf $=$ $128.8 / 120$. As the reactor is a key for current and future neutrino projects, validation by multiple experiment is essential. The precision is currently limited by Statistics. New $\theta_{13}$ results with $\mathrm{Gd}+\mathrm{H}$ analysis will give better precision. Other interesting physics programs are also underway.

\section{References}

[1] Y. Abe et al., Phys. Rev. Lett. 108, 131801 (2012).

[2] F. P. An et al., Phys. Rev. Lett. 108, 171803 (2012).

[3] J. K. Ahn et al., Phys. Rev. Lett. 108, 191802 (2012).

[4] Y. Abe et al., Phys. Rev. D 86, 052008 (2012).

[5] Y. Abe et al., Phys. Lett. B 723, 66 (2013).

[6] Y. Abe et al., JHEP 10, 086 (2014). 